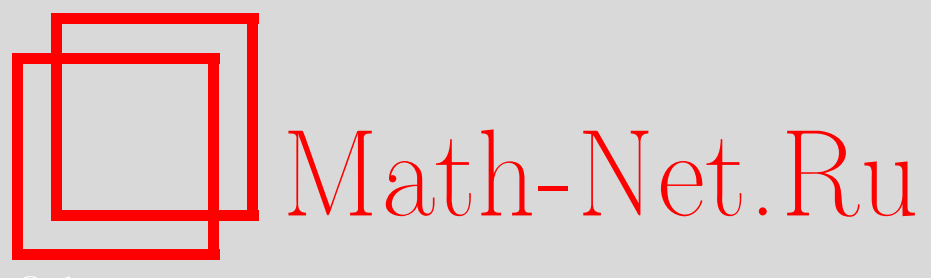

А. А. Давыдов, Л. Чинь Тхи Зиеп, Нормальные формы семейств линейных уравнений смешанного типа вблизи нерезонансных сложенных особых точек, УМН, 2010, том 65, выпуск 5, 189-190

DOI: https://doi.org/10.4213/rm9386

Использование Общероссийского математического портала Math-Net.Ru подразумевает, что вы прочитали и согласны с пользовательским соглашением http: //www . mathnet.ru/rus/agreement

Параметры загрузки:

IP: 54.166 .219 .16

26 апреля 2023 г., $17: 27: 34$

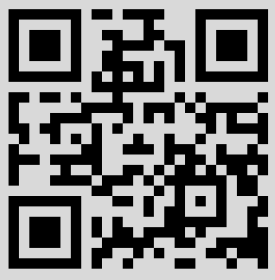




\title{
Нормальные формы семейств линейных уравнений смешанного типа вблизи нерезонансных сложенных особых точек
}

\author{
А. А. Давыдов, Л. Чинь Тхи Зиеп
}

В точке плоскости характеристическое направление $d x: d y$ линейного уравнения второго порядка с частными производными - это решение уравнения

$$
a(x, y) d y^{2}-2 b(x, y) d x d y+c(x, y) d x^{2}=0,
$$

где $x, y$ - координаты на плоскости, а $a, b, c$ - коэффициенты главного символа уравнения, которые мы предполагаем достаточно гладкими. В точке плоскости есть 2,1 либо 0 вещественных характеристических направлений, если значение дискриминанma $D:=b^{2}-a c$ в этой точке $>0,=0$ либо $<0$ соответственно. Характеристики интегральные кривые поля этих направлений - играют важную роль в теории дифференциальных уравнений (см., например, [1], [2]), поэтому классификация уравнений характеристик - важная прикладная задача, постановка которой восходит к концу 19-го века [3]. С начала 20-го века эта классификация (с точностью до гладких замен координат и умножения на гладкую функцию) включает уравнения $d y^{2}+d x^{2}=0$, $d y^{2}-d x^{2}=0$ и $d y^{2}-x d x^{2}=0$, к которым уравнение (1) приводится вблизи точки области эллиптичности $(D<0)$, области гиперболичности $(D>0)$ и типичной точки линии смены типа (где $D=0, d D \neq 0$, а характеристическое направление трансверсально границе области) соответственно [4], [2]. Классификация типичных особенностей здесь была завершена в конце 20-го века [5]-[7], и в ней ключевую роль сыграла теорема редукции, сводящая теорию нормальных форм сети характеристик вблизи точек их касания с линией смены типа к теории нормальных форм векторных полей на плоскости и согласованных с ними инволюций. Здесь мы доказываем эту теорему для случая $C^{\infty}$-семейств уравнений (1) с конечномерным параметром $\varepsilon$ и, используя также результаты [8], получаем конечно гладкие нормальные формы для сети характеристик вблизи нерезонансных сложенных особых точек уравнения (1).

Предполагается, что левая часть уравнения (1) при заданном значении параметра (выбираемого нами за нуль) невырождена, поэтому это уравнение задает гладкую поверхность уравнения в пространстве направлений на плоскости (с переменными $x, y$, $d x: d y)$, а складывание уравнения - ограничение на эту поверхность проекции вдоль оси направлений (т. е. вертикальной оси) $(x, y, d x: d y) \mapsto(x, y)$ - имеет лишь критические точки типа "складка Уитни". Эти критические точки образуют криминанту уравнения. На этой поверхности определена гладкая инволюиия складывания - переставляющая точки с одинаковыми образами при складывании уравнения [5].

Поле характеристических направлений уравнения поднимается до гладкого поля направлений на этой поверхности, вообе говоря, с особыми точками. Это поле в точке поверхности задается пересечением касательной плоскости и контактной плоскости (задаваемой характеристическим направлением и вертикальным). Отметим, что особые точки поля направлений уравнения - это в точности точки криминанты, где эти две плоскости одинаковы. В остальных точках криминанты это поле вертикально и является собственным направлением производной инволюции складывания с собственным числом -1 . В этой ситуации теорема редукции сводит теорию нормальных форм сети характеристик вблизи точки ее касания с линией смены типа к теории нормальных форм пары (поле направлений, инволюция складывания) вблизи особой точки последнего поля на поверхности уравнения.

Векторное поле и дифференцируемая инволюция $\sigma$ с линией неподвижных точек называются согласованными в точке этой линии, если вблизи этой точки определитель матрицы, составленной из значений этого поля и его образа при этой инволюции,

Первый автор поддержан АВЦП РНПВШ (проект 2.1.1/5568) и программой "Математическая теория управления" Президиума РАН. 
имеет нуль второго порядка на этой линии. Поле направлений и такая же инволюция согласованы в точке этой линии, если это поле можно задать векторным полем, согласованным с этой инволюцией в этой точке. Аналогично вводится понятие согласованности и для дифференцируемых семейств пар полей и инволюций с одним и тем же параметром. Для типичного уравнения (1) инволюция складывания и поле направлений уравнения на его поверхности согласованы в любой точке криминанты.

Два ростка объектов одной природы (отображений, кривых, ... ) $C^{k}$-эквивалентны вдоль дифференцируемого векторного поля $v$ (или $C_{v}^{k}$-эквивалентны), если они переводятся один в другой ростком $C^{k}$-диффеоморфизма, переводящим локально фазовые кривые поля $v$ в себя. Для ростков семейств объектов $C_{v}^{k}$-эквивалентность это $C^{k}$-диффеоморфизм, сохраняющий естественное расслоение над пространством параметра семейства и переводящий фазовые кривые поля $(v, \dot{\varepsilon}=0)$ в себя; $C_{v}^{k}$-эквивалентность сильная, если она, к тому же, сохраняет параметр.

Теорема 1. Для $C^{\infty}$-семейства $v$ векторных полей ростки в нуле двух $C^{\infty}$-семейств инволючий $\sigma_{1}$ и $\sigma_{2}$ с одним и тем же конечномерным параметром в сильно $C_{v}^{\infty}$-эквивалентны, если эти семейства инволюиий имеют одну и ту же поверхность неподвижных точек, проходлщую через нуль, и согласованы с $v$ в нуле, а векторное поле $(v, \dot{\varepsilon}=0)$ трансверсально этой поверхности почти всюду вблизи нуля.

Из теоремы 1 и полученных в [6] конечно гладких нормальных форм вытекает

Теорема 2. Для любого натурального числа $r$ конечно параметрическая $C^{\infty}$-деформачия ростка уравнения (1) в его нерезонансной сложенной особой точке приводится к ростку в нуле деформачии $d y^{2}+\left(k(\varepsilon) x^{2}-y\right) d x^{2}=0$, где $\varepsilon-$ параметр деформачии, а $k$ - некоторая функиия, подходящим выбором расслоенных над параметром $C^{r}$-координат и домножением уравнения на не обращающуюся в нуль функиию от $x, y$ и

Для сложенных узлов и фокусов можно взять $r=\infty$. Резонансность невырожденной особой точки уравнения (1) определяется как резонансность соответствующей невырожденной особой точки дифференцируемого векторного поля, задающего поле направлений этого уравнения на его поверхности. Функция $k$ вычисляется по собственным числам линеаризации поднятого поля в особой точке так же, как в [5]-[7].

В [9] дан обзор результатов по локальным нормальным формам уравнения (1).

\section{Список литературы}

[1] А.Г. Кузьмин, Неклассические уравнения смешанного типа и их приложения $к$ газодинамике, Изд-во Ленинградского ун-та, Л., 1990, 207 с. [2] С. Л. Соболев, Уравнения математической физики, Наука, 1966. [3] C. Weierstrass, C. Hermite, G. Mittag-Leffler, Acta Math., 7 (1885), I-VI. [4] M. Cibrario, Ist. Lombardo, Rend., II Ser., 65 (1932), 889-906. [5] А. А. Давыдов, Функи. анализ и его прил., 19:2 (1985), 1-10. [6] A. A. Davydov, Qualitative theory of control systems, Transl. Math. Monogr., 141, Amer. Math. Soc., Providence, RI, 1994, viii+147 pp. [7] А. А. Давыдов, Э. Росалес-Гонсалес, Докл. РАН, 350:2 (1996), 151-154. [8] Ю. С. Ильяшенко, С. Ю. Яковенко, УМН, 46:1 (1991), 3-39. [9] F. Tari, "Pairs of foliations on surfaces", Real and complex singularities, London Math. Soc. Lecture Note Ser., 380, Cambridge Univ. Press, Cambridge, 2010 (to appear).

\section{А. А. Давыдов (А. А. Davydov)}

Владимирский государственный университет

E-mail: davydov@vlsu.ru, davydov@iiasa.ac.at

\section{Л. Чинь Тхи Зиеп (L. Trinh Thi Diep)}

Владимирский государственный университет

E-mail: dieplinhsptn@yahoo.com.vn
Представлено В. М. Закалюкиным Принято редколлегией 17.09.2010 